bildet für das Individuum die ,Krise der Identität"“" (Hall I994: I 8of.) In dieser Krise mag der Wandel selbst mitunter als das neue Kontinuierliche erscheinen, als eine beständige Trans-Formation des Gewohnten.

\title{
5. Zeichen: Symbol und Ritual
}

Daß der Mensch ein animal symbolicum sei, lehrt uns heute vielleicht nicht mehr das humanistische Bildungsgut der Lateinstunde, dafür tun dies um so intensiver die Reklamebilder der Alltagswelt. Mit der ästhetischen und semantischen Reduktion ihrer Botschaften auf wenige signifikante Zeichen und Gesten bestätigen sie uns, daß menschliche Welten stets Zeichen- und Symbolwelten waren und sind. Symbole und Rituale fungieren - um die Computersprache $\mathrm{zu}$ benutzen - als die "Steuerzeichen“ unserer Kultur. Sie bilden die großen Verständigungskodes sozialer Beziehungen, wobei die Symbole gewissermaßen den Bedeutungskode repräsentieren, die Rituale dagegen den Handlungskode. Und es gibt eine weitverbreitete Auffassung, die man in dieser Radikalität allerdings nicht teilen muß, wonach die Kulturwissenschaften sich ausschließlich der Beschreibung und Interpretation dieser menschlichen Zeichensysteme zu widmen haben.

Etymologisch betrachtet, stammt unser moderner Symbolbegriff vom griechischen Wort symballein ab, das „zusammenfügen“, ,zusammenwerfen" bedeutet. In der griechischen Mythologie steht dies als Sinnbild für ein Zusammenfügen von zwei Teilen, die einst eins waren, jedoch getrennt worden sind. Es ist das Motiv der Münze oder Schale, die in zwei Teile zerbrochen wird, um nach einer möglicherweise langen Trennung als untrügliches Erkennungszeichen der Identität des Träger oder Überbringers zu dienen. Der Begriff Ritual wiederum ist vom lateinischen ritus abgeleitet, meint also einen „Brauch“ im Sinne eines präzise geformten und tradierten Verhaltensmusters, enthält aber auch die Bedeutung von „In-GebrauchSein“. Beide Begriffe beziehen sich wohl ursprünglich auf eine religiöse Grundtextur der Kultur, in deren Webmustern bestimmte kultische Zeichen und Bereiche markiert waren, die eine zentrale Rolle spielten für das soziale Selbstverständnis und die Weltbilder von Menschengruppen. Religiös meint in diesem Sinne weniger Glaubensformen als vielmehr ein weltzugewandtes Werte- und Ordnungssystem, das Respekt vor den Regeln sozialen Zusammenlebens vermittelte und diese zum Kern des gesellschaftlichen Konsensus erklärte: zu etwas Heiligem, zum höchsten sozialen Wert. Um diesen Respekt und damit letztlich Gesellschaft zu sichern, dienten solche 
religiösen Kulte der feierlichen Bestätigung solcher Übereinkünfte, wobei Symbole als verkörpernde Zeichen wirkten und Rituale als verbindende Handlungen. Symbol und Ritual als kultische Elemente beschworen insofern communitas, Gemeinschaft.

Wie jede andere Religionsgeschichte bietet dafür auch die christliche zahllose einschlägige Beispiele: das Kreuz etwa als „Zeichen Gottes“ und damit als Symbol der christlichen „Weltanschauungsgemeinschaft" oder der Gottesdienst als rituelle, kollektive Bestätigung der Gültigkeit dieses Symbols und darüber hinausweisend der christlichen Ethik und Moral. So wurde das Kreuzzeichen zum gegenseitigen Identifikationssymbol der „Christenmenschen“; Orte unter dem Kreuz wie Kirchen und Friedhöfe bildeten tabuisierte Schutzräume für Arme und Verfolgte; politische und wirtschaftliche Geschäfte wurden durch rituelle Handlungen unter dem Kreuz bezeugt, also durch das Symbol bekräftigt und geschützt. Ähnliche Funktionen von Symbol und Ritual galten wohl für alle religiösen Kulturen. Daher lag es zum Beispiel für die Völkerkunde stets nahe, sich schwerpunktmäßig mit religiösen und magischen Praktiken in unterschiedlichen Gesellschaften zu beschäftigen, da sich hier erhellende Aufschlüsse über soziale Weltbilder, symbolische Tabus und kulturelle Ordnungen finden lassen mußten. ${ }^{42}$ Aber auch die symbolische Bedeutung und rituelle Funktion von Geschenk und Gabe etwa - als eine „Pflicht des Gebens und Nehmens“ - wurde in ,archaischen“" Gesellschaften immer als ein zentrales Religions- und Loyalitätsprinzip betrachtet. ${ }^{43}$

\section{Zeichentheorien}

Die Verwendung von Symbolen und Ritualen hat sich allerdings früh schon aus diesem engeren religiösen Ursprungsbereich gelöst. Auch die Grenzen ihres zweiten klassischen Anwendungsbereichs, jenem von Macht und Herrschaft, hat sie längst überschritten, in dem Symbole und Rituale wesentliche Insignien politischer Herrschaftsbestätigung bildeten. Symbole und Rituale sind in der modernen Welt inzwischen ständige Begleiter, ja Gestalter des Alltagslebens geworden. Dabei wird man nicht sagen können, daß sie sich in den Alltag ausgebreitet haben. Vielmehr hat sich offenbar unser Symbolund Ritualverständnis so erweitert, daß wir es inzwischen auf vielfältige kodifizierte Interaktionsregelungen anwenden, wenn sie nur eine gewisse Zeichenhaftigkeit und Formenfestigkeit aufweisen $(\mathrm{Bu}-$ kow I984; Goffman I99I). Daß damit eine Tendenz besteht, fast alles von der Arbeit bis zur Freizeit, vom Essen bis zur Liebe in symbolischen und rituellen Handlungen gefaßt zu sehen, birgt natürlich die Gefahr einer Entwertung der analytischen Aussagekraft dieser Begrif- 
fe in sich. Gleichwohl ist damit der Erkenntnis Rechnung getragen, daß Kultur in ihrer Mikroorganisation tatsächlich symbolisch und rituell durchformt ist, daß es stets um jenen hochdifferenzierten Kode von Zeichen, Mustern und Bedeutungen geht, der im Alltagsleben umständliche Handlungen und Erklärungen auf ihr Wesentliches reduzieren hilft. ${ }^{44}$

So läßt sich Kultur als ein expressives Zeichen- und Bedeutungssystem charakterisieren, in dem die Symbole und Rituale wie Computerchips wirken, die jeweils eine komplette Informationsdatei in sich speichern. Umgekehrt weist jede soziale Handlung bei genauerer Betrachtung ihrer kulturellen Formgestaltung eine Vielzahl kleinster Zeichen und Muster auf, deren komplexe Bedeutungshaftigkeit wir meist erst dann erkennen, wenn wir sie nicht verstehen, wenn sie uns nicht ,,selbstverständlich“, sondern „fremd“ ist. Das kulturell Andere wird uns gerade in solchen Situationen und an solchen Orten deutlich, wo wir den kulturellen Kode von Symbolen und Ritualen nicht entschlüsseln, also auch nicht selbst befolgen können: bei Begrüßungen, bei Mahlzeiten, bei feierlichen Anlässen, bei religiösen Zeremonien in unvertrauten kulturellen Zusammenhängen. Und diese Erfahrung eines Andersseins tritt keineswegs nur beim Kontakt mit Menschen aus fernen Ländern auf, sondern kann uns bereits beim Betreten des „falschen“ Lokals, Geschäfts oder Hörsaals gleich um die Ecke betreffen oder in der Beziehung zwischen den Geschlechtern.

Das Spektrum dieser vielfältigen Kulturzeichen ist daher längst in eine generelle Zeichentheorie eingebunden, in die Semiotik, die sich vor allem mit sprachlichen, bildlichen und gestischen Zeichen beschäftigt und die - zurückgreifend auf Arbeiten etwa des englischen Philosophen John Locke (I632-I704) oder des Schweizer Sprachwissenschaftlers Ferdinand de Saussure (I857-I9I3) - bereits nach der Jahrhundertwende entwickelt wurde. Ausgehend von linguistischen, d.h. sprachwissenschaftlichen Überlegungen, entwarf man eine logische Systematik der Zeichen, die sich in drei Dimensionen gliedern ließ: in die Syntax als die Beziehung sprachlicher Zeichen zueinander, in die Semantik als Beziehung zwischen den sprachlichen Zeichen und dem gemeinten Objekt sowie in die Pragmatik als Beziehung zwischen Sprecher und Hörer bzw. Leser oder Betrachter. ${ }^{45}$

Schon aus dieser Systematik wird deutlich, daß es in der Semiotik stets um Bezeichnungen und Beziehungen geht, um Benennungen und Bedeutungen, um die Regeln einer Interpretation des Geschriebenen, Gesagten und Gemeinten - damit also immer auch um Beschreibungsdimensionen und Deutungsprobleme von Kultur. Die ursprünglich linguistischen Überlegungen sind daher verallgemeinert worden zu Theorien einer Kultursemiotik, einer Lehre von den Kul- 
turzeichen. Sie geht - sehr verkürzt gesagt - davon aus, daß jedes Wort, jeder Satz nicht nur den Gehalt des Gesagten, sondern darüber hinaus einen „Überschuß“ an Bedeutetem enthält, daß sich seine Semantik also annähernd vollständig nur über die Erschließung seines Bedeutungskontextes verstehen läßt. So ist leicht nachvollziehbar, daß die simple Bemerkung ,Heiß heute!“ in einem Universitätsseminar eine etwas andere Bedeutung besitzt als auf einer Baustelle. Selbst wenn die Bemerkung an beiden Orten ohne jeden weiteren Zusatz gewechselt worden sein sollte, verweist allein schon die unterschiedliche physische und soziale Arbeitssituation auf unterschiedliche Semantiken.

Der Kontext also verleiht spezifische Bedeutungen, denn die sozialen Räume und die Zuhörenden sind es, die dem Gesagten ihre Deutungen hinzugeben: Sie hören den Satz bereits selbst ,interpretierend". Diese Interpretationskontexte gestalten sich noch sehr viel komplizierter, wenn es nicht um einen simplen Satz, sondern um ein bereits bedeutungskomplexes Symbol und Ritual geht. Aufgabe der Kultursemiotik ist es daher, diese Komplexität zu entschlüsseln, d.h. die einzelnen Elemente eines Symbol- und Zeichensystems in ihrem jeweiligen Bedeutungszusammenhang zu rekonstruieren und damit gleichsam die Regeln der ,,kulturellen Grammatik“ offenzulegen.

\section{Rites de passage}

Man hat in der Völkerkunde wie in der Volkskunde immer wieder versucht, Symbole und Rituale systematisch in Bedeutungsgruppen oder Praxisfeldern zusammenzufassen, um einen besseren Überblick über dieses komplizierte Formen- und Regelwerk der Kultur zu erhalten. ${ }^{46}$ Konsequent sind diese Überlegungen nicht zu Ende geführt worden, weil die Welt der Symbole sich als zu vielfältig und vice versa deren Zuordnung zu einem einzigen Zweck sich als zu einfältig erwiesen hat. Immerhin konnten einige dieser Gruppenbildungen doch eine gewisse Plausibilität erreichen, weil sie sehr nahe an kulturellen Praxisformen konzipiert waren. ${ }^{47}$

Ein solches Feld, auf das im Zusammenhang ethnologischer Symbol- und Ritualforschung näher eingegangen werden muß, ist mit der Überschrift „Rituale des Übergangs“, rites de passage, versehen. Dieser Begriff ist wesentlich geprägt von dem französischen Ethnographen Arnold van Gennep (I873-I957), der nicht als universitärer Forscher, sondern als Übersetzer und Journalist freiberuflich tätig war, dennoch als Mitbegründer einer modernen französischen Ethnologie gelten muß (Chiva I987: 3 If.). Van Gennep beschäftigte sich insbesondere mit „Grenzsituationen“ im Leben des einzelnen wie der Gesellschaft, und er stieß überall auf solche Grenzen und auf de- 
ren entsprechende Markierung in Form von Symbolen und Ritualen. Jahres- und Lebenszeiten, Wechsel zwischen Berufen, Schichten, Religionen und Altersstufen setzen Grenzen, bedeuten Trennungen, an denen sich etwas verändert, etwas anderes oder Neues beginnt. Damit Gesellschaft aber nicht nur aus solchen Grenzen besteht, müssen Übergänge vorhanden sein, die den Wechsel und Wandel ermöglichen.

Van Genneps Hypothese lautet, daß diese Übergänge gleichsam die Schwachstellen aller Gesellschaften seien, weil Grenzen zugleich auch Ordnung bedeuten. Wer Grenzen überschreitet, begibt sich aus einer Ordnung heraus, muß also gleichzeitig in eine andere Ordnung eingebunden werden, um nicht das gesellschaftliche Gesamtsystem zu stören oder es zu zerstören. Alle Gesellschaften versuchten daher, diese Grenzüberschreitungen und Übergänge zu kontrollieren, indem sie rituelle und symbolische Festlegungen trafen, wie das Verlassen der alten und das Erreichen der neuen Position zu bewerkstelligen sei. Es sind die rites de passage, die diesen Wechsel regeln - seien es lebensgeschichtliche oder soziale Wechsel. Sie steuern die Modalitäten der Veränderung für den Betroffenen, sie bestimmen zugleich aber auch die Modalitäten des Umgangs damit für seine soziale Umgebung. „In jeder Gesellschaft besteht das Leben eines Individuums darin, nacheinander von einer Altersstufe zur nächsten und von einer Tätigkeit zur anderen überzuwechseln. Wo immer zwischen Altersund Tätigkeitsgruppen unterschieden wird, ist der Übergang von einer Gruppe zur anderen von speziellen Handlungen begleitet, wie sie etwa der Lehre bei unseren Handwerksberufen entsprechen. (...) Jede Veränderung im Leben eines Individuums erfordert teils profane, teils sakrale Aktionen und Reaktionen, die reglementiert und überwacht werden müssen, damit die Gesellschaft als Ganzes weder in Konflikt gerät, noch Schaden nimmt. Es ist das Leben selbst, das die Übergänge von einer Gruppe zur anderen und von einer sozialen Situation zu anderen notwendig macht. Das Leben eines Menschen besteht somit in einer Folge von Etappen, deren End- und Anfangsphasen einander ähnlich sind: Geburt, soziale Pubertät, Elternschaft, Aufstieg in eine höhere Klasse, Tätigkeit, Spezialisierung. Zu jedem dieser Ereignisse gehören Zeremonien, deren Ziel identisch ist: Das Individuum aus einer genau definierten Situation in eine andere, ebenso genau definierte hinüberzuführen." (Gennep I986: I 5, 2 I)

So argumentiert van Gennep, in diesen Ritualen auch eine zyklische, regenerative Erneuerungskraft aller Kulturen vermutend. Er schlägt daher vor, die allgemeine Kategorie der Übergangsriten zu untergliedern in Trennungsriten, Umwandlungsriten und Angliederungsriten. Und er versucht neben den Funktionen auch die Formen zu gliedern, nämlich in räumliche, soziale und biographische Über- 
gangsriten. Mit diesen Modellen macht er deutlich, wie sehr diese Rituale in der Tat Scharniere der kulturellen Funktionssysteme jeder Gesellschaft sind, da sie das Individuum in seine soziale Bezugsgruppe integrieren, den Umgang mit Gefühlen und Beziehungen regeln und dadurch letztlich Identität sichern. Den Sinn seiner Einteilungen macht er am Beispiel der Heirat deutlich: „Die Heirat stellt den wichtigsten Übergang von einer sozialen Kategorie zur anderen dar, weil sie - für einen Ehepartner zumindest - einen Familien-, Klan-, Dorf- oder Stammeswechsel zur Folge hat. Manchmal bezieht das frisch verheiratete Paar auch ein neues Haus. Ein solcher Wohnortswechsel wird in den Hochzeitszeremonien durch Trennungsriten zum Ausdruck gebracht, die hauptsächlich auf den räumlichen Übergang Bezug nehmen. Da so viele Gruppen von der sozialen Vereinigung zweier ihrer Mitglieder betroffen sind, ist es andererseits verständlich, daß die Übergangszeit hier eine besondere Bedeutung erlangt. Sie wird gewöhnlich als Verlobungszeit bezeichnet. Bei einer Vielzahl von Völkern bildet die Verlobung einen besonderen und selbständigen Teil der Hochzeitszeremonien. Sie umfaßt Trennungsund Schwellen- bzw. Umwandlungsriten und endet mit Riten, die entweder eine vorläufige Angliederung an die neue Umwelt oder eine Trennung von der selbständigen Übergangsphase gewährleisten. Dann folgen die Hochzeitszeremonien, die hauptsächlich aus Riten bestehen, die endgültig an die neue Umgebung angliedern." (Gennep I986: I I4f.)

Van Genneps Beschreibung dieser dreistufigen Heiratsriten entspricht wohl nicht mehr in jeder Hinsicht heute gültiger Praxis. Doch lassen sich Restformen dieser rituellen und symbolischen Regeln durchaus noch im Ablauf moderner Hochzeitsfeiern erkennen, vor allem dort, wo in einem entsprechend engen verwandtschaftlichen, freundschaftlichen oder nachbarschaftlichen Umfeld das Zeremonielle gefördert und betont wird. Denn in ,starken“ Beziehungssystemen werden Rituale meist auch deshalb aktiver überliefert und intensiver praktiziert, weil sie diese Beziehungssysteme ihrerseits wiederum stärken.

Ähnlich verhält es sich mit einem zweiten Typus, den van Gennep in seinen Studien unter dem Begriff der Initiationsriten vorstellt, also der rituellen Einführung in neue Alters- oder Berufsrollen. Er verweist dabei vor allem auf das Beispiel afrikanischer Gesellschaften, in denen - teils bis heute - die rituelle Einführung junger Frauen und Männer in den Erwachsenenstatus verbunden ist mit körperlichen und seelischen Prüfungen wie Fastenzeiten, Gemeinschaftsritualen oder der inzwischen vieldiskutierten Beschneidung der Sexualorgane (Douglas 1986). Auch diese gemeinsame rituelle Bewältigung eines Rollenwechsels in einer Alterskohorte findet bei uns noch in modifi- 
zierter Form ihre Entsprechungen etwa in den rituellen Aufnahmeverfahren männlicher Jugendgruppen. Mutproben, rituelles Trinken, das Tragen von Gruppenabzeichen und auch Gewaltriten verkörpern in diesen Peer-group-Strukturen überlieferte, zum Teil selbst entworfene Initiationsriten, die ein „Gemeinschaftsgefühl“ symbolisch signalisieren wie emotional herstellen sollen.

Das bereits erwähnte Center for Contemporary Cultural Studies in Birmingham hat seine Cultural Studies in den siebziger Jahren auf diese modernen Jugendriten konzentriert, um daran zu analysieren, wie in einer modernen Gesellschaft Prozesse der sozialen Gruppenbildung, aber auch der Klassenabgrenzung symbolisch und rituell strukturiert sind (Clarke u.a. I979). In der Tat weist gerade die heutige Jugendkultur eine extreme Vielfalt der Mode- und Musikrichtungen, der Sport- und Freizeitstile auf, in denen sich ästhetische Praxis als sozial hochdifferenziert, symbolisch geformt und rituell organisiert erweist. Frisuren, Piercing, Tätowierungen, Abzeichen, Schuhe, Kleidungsmarken verraten dem Eingeweihten genau, welche Gruppenzuordnung damit beabsichtigt ist. Es ist ein permanenter Prozeß des Sich-Zuordnens und Sich-Abgrenzens, der offenbar in immer schnelleren Rhythmen und Wechseln erfolgt. Und weil sie nicht nur die eigene Gruppensymbolik kennen, sondern auch die der anderen, sind manche der Jugendlichen gewiß bessere ethnographische Kenner dieser Szene, als es die meisten professionellen Jugendkulturforscher je sein können. Gerade in ihrer extremen Ausdifferenzierung erscheint die Praxis der symbolischen Gruppenstilisierung als ein typisches Phänomen der Moderne. Dabei handelt es sich aber - mit van Gennep - um eines der ältesten Muster kultureller Selbststilisierung und Abgrenzung, das quer durch die Kulturen und Zeiten auftritt.

\section{Symbolisches Krisenmanagement}

Van Genneps Überlegungen zu den rites de passage sind heute nicht unumstritten, weil seine Theorie von einer Art Naturgesetzlichkeit dieser besonderen Ritualform ausgeht und sie als symbolische Struktur kultureller Praxis vielfach aus ihren sozialen Kontexten herauslöst. Auch erfahren wir wenig über soziale Wandlungsprozesse, dafür um so mehr über oft recht unbesehen behauptete Gemeinschaftsund Gruppenidentitäten. Dennoch bleiben van Genneps generelle Beobachtungen der "Schwellensituationen“ als Prozesse sozialer Desintegration und einer nachfolgenden Reintegration durch „,̈bergangsriten“ als kulturelle Bewältigungsstrategien von zentraler Bedeutung auch für moderne Lebenswelten. ${ }^{48}$

Diese Überlegungen aufgreifend, hat der englische Sozialanthropologe Victor Turner seit den I960er Jahren versucht, van Genneps 
Theorien insofern weiterzuentwickeln, als er die Problematik der Übergangssituationen und Übergangsriten auf ganze Gesellschaften anwendet. Er fragt danach, wie Gesellschaften in Umbruchs- und Krisensituationen diesen Übergang kulturell organisieren, wie sie vor allem die gefährliche ,mittlere Phase“ zwischen dem Verlassen des alten Status und dem Erreichen der neuen Ordnung bewältigen. Zur Kennzeichnung dieser Situation benutzt er den Begriff der „Liminalität“, also der Schwellenhaftigkeit, in der dann eine „offene Gesellschaft" entsteht, die ihre neue Position erst suchen und finden muß. Es ist ein Suchen nach neuen gemeinsamen Nennern, nach einem Zustand der „Communitas“, also der Gemeinschaft, die sich in der Suche nach neuen Horizonten herstellen soll. Initiatoren solcher kommunitären Prozesse sind in der europäischen Geschichte etwa religiöse Ordensbewegungen oder politische Erneuerungsbewegungen. Turner rechnet in der jüngsten Zeit auch soziale Bewegungen wie Flower-Power oder Hippies dazu, die ebenfalls gesellschaftliche Erneuerung wollten und sie auch bewirkten. Zugleich sind diese Bewegungen Indikatoren für soziale Krisen, insofern sie der Gesellschaft deren Erstarrtheit deutlich machen und demgegenüber zur Öffnung und zur Suche auffordern. Suche aber bedeutet Orientierungsprobleme, Unsicherheit, Angst. Deshalb entwickeln Gesellschaften in dieser Situation der „Liminalität“ ein besonders großes Bedürfnis nach symbolischen und rituellen Orientierungszeichen, die den neuen Weg bezeichnen, bestätigen und sichern und die dadurch auch manche Markierung des alten Weges überdecken (Turner I989b).

Turners Konzept ist nur ein Beispiel dafür, wie nützlich ethnologische Theorien zur symbolischen und rituellen Struktur der Kultur sein können, um gesellschaftliche Rahmensituationen und kulturelle Praktiken in einen analytischen Zusammenhang zu bringen. Dazu ließe sich eine ganze Reihe anderer einschlägiger Theorien nennen, die mit Namen wie dem des amerikanischen Kulturanthropologen Clifford Geertz oder des französischen Kultursoziologen und Ethnologen Pierre Bourdieu verbunden sind und die zum Teil in den vorangegangenen Kapiteln angeschnitten wurden. Doch möchte ich hier auf deren Erörterung verzichten und statt dessen abschließend danach fragen, inwieweit sich Turners Überlegungen sinnvoll auch auf Situationen anwenden lassen wie die, in der sich die deutsche Gesellschaft in den I990er Jahren befindet. Man könnte mit Turner fragen: Stehen wir heute, zu Beginn des als „nachmodern“ und „postsozialistisch“ apostrophierten Zeitalters, in diesem ersten Jahrzehnt nach dem Zusammenbruch des politischen Systems der DDR und nach der deutschen Vereinigung, in einer derartigen gesellschaftlichen Schwellensituation? Befinden wir uns in einer Phase des 
Übergangs, in der die gesellschaftlichen Wertehorizonte und Selbstbilder gleichzeitig durch ein ,nicht mehr“ wie durch ein ,noch nicht" gekennzeichnet sind?

\section{Europäische Übergänge}

Für diese Annahme läßt sich eine Anzahl von Indizien ins Feld führen, deren Schlagworte uns allen geläufig sind: der globale Hintergrund ökonomischer wie ökologischer Krisenphänomene, die schwierige Orientierung zwischen europäischen und nationalen Horizonten, die durch die deutsche Vereinigung wie den Zusammenbruch des Sozialismus in Osteuropa neu aufgeworfenen Fragen nach dem langen Schatten von Nationalsozialismus und Holocaust, die komplizierten wechselseitigen Bilder von Ostdeutschen und Westdeutschen, die sozialen und psychischen Folgen der Massenarbeitslosigkeit oder die Probleme einer Einwanderungsgesellschaft, die sich mit dem politischen wie kulturellen Bekenntnis dazu schwertut. All diese Stichworte markieren komplizierte gesellschaftliche Veränderungen und Erfahrungslagen, die nachhaltig in unsere Alltagswelten hineinwirken, deren Horizonte aufbrechen und bis in die Lebensläufe hinein Erfahrungen und Erwartungen, Pläne und Hoffnungen verunsichern.

Gegen dieses Krisenszenario mag man einwenden, daß sich diese Verunsicherung vielfach auch als ein mediales Phänomen erklären ließe, daß nur bestimmte Gruppen unmittelbar davon betroffen seien, die sich etwa im Bereich der Dauerarbeitslosigkeit befinden, oder nur bestimmte Regionen, die in Ostdeutschland vor allem noch in einer strukturellen Übergangssituation stehen. Demnach beträfe diese Schwellensituation nur bestimmte gesellschaftliche Segmente, nicht das Ganze. Und wenn man weite Teile der gesellschaftlichen Landschaft in Westdeutschland betrachtet, kann man in der Tat den Eindruck gewinnen, daß dort versucht wird, Krisengefühle in dieser Weise wegzudrängen. $\mathrm{Ob}$ mit oder ohne Erfolg: Die labile Situation „auf der Schwelle“ wird jedenfalls kurzerhand den Ostdeutschen zugewiesen.

Nun braucht man sich über den Befund gar nicht einig zu sein, um feststellen zu können, daß ein wesentliches Charakteristikum der „Liminalität"“ auf die deutsche Befindlichkeit der Gegenwart zutrifft: ein - verglichen noch mit den I970er und 8oer Jahren - deutlich anderer und wesentlich erhöhter Symbol- und Ritualbedarf. Welche Wertehorizonte unseren gesellschaftlichen Raum überwölben können, welche Bilder Deutschlands und Deutsch-Seins nach innen wie nach außen zu vermitteln sind, das wird weniger auf der Ebene gesellschaftspolitischer Entwürfe verhandelt als vielmehr durch die Pro- 
duktion symbolischer Bilder und Zeichen, ritueller Handlungen und Gesten kommentiert. Diese Symbolproduktion vollzieht sich in einem ungeheuer breiten Themenspektrum, das von den inszenatorischen Höhen nationaler Geschichts-, Feier- und Denkmalskultur hinunterreicht bis in die Niederungen individueller Lebens- und Freizeitstile, die nicht nur einfach ,gelebt“, sondern ebenfalls inszeniert und stilisiert daherkommen müssen. Denkmalswettbewerbe, Gedenktage, Museen, Repräsentationsarchitektur stehen so neben Love-Parades, Sportevents, Golfausrüstung, gestylten Trabis, Designerkleidung: Wir sind nicht nur von öffentlichen Symbolen und Zeichen umstellt, sondern wir umstellen unseren Alltag selbst mit immer dichteren Symbolkulissen, um uns darin unserer Räume, Wege und Rollen zu versichern. ${ }^{49}$

Dabei nimmt sich die deutsche Situation in vieler Hinsicht symptomatisch aus für ein europäisches Szenario, denn sie spiegelt westwie osteuropäische Krisengefühle gleichermaßen wider. Besonders die osteuropäischen Gesellschaften bedürfen gegenwärtig offenbar einer intensiven „Politik der Symbole“, die jenes Vakuum auszufüllen versucht, das der Zusammenbruch der sozialistischen Deutungs- und Zeichenwelt hinterlassen hat, die aber auch neue, nationalistische und ethnische Vergemeinschaftungen zu erzeugen versucht (Niedermüller I994), indem sie alte Mythen und Riten des vorsozialistischen Nationalismus revitalisiert. Natürlich liefern ihnen west- und nordeuropäische Gesellschaften, die in commemorative ceremonies ihre nationalen Gründungsfeiern und Feste inszenieren, auch dafür längst Vorbilder und Modelle. ${ }^{50}$

Wenn man diese kulturelle Symptomatik ernst nimmt, dann spricht sie doch eine deutliche Sprache der Verunsicherung über „das Eigene“ und „das Andere“, über individuelle wie kollektive Identitäten. Dann könnte umgekehrt aber auch eine systematische Analyse solcher Symbolproduktion und Symbolpraxis wichtige Einblicke in die Suchbewegungen und die Experimentierfelder europäischer Gesellschaft eröffnen, die - auf einer „Schwelle“ ihrer eigenen Geschichte wie der Weltgeschichte stehend - offenbar nicht so recht weiß, ob sie nach vorn oder zurückblicken soll. ${ }^{51}$ Denn: „Das ethische Paradox des postmodernen Zustands besteht darin, den gesellschaftlichen Subjekten die Vollständigkeit moralischer Entscheidung und Verantwortung zurückzugeben und ihnen gleichzeitig die Sicherheit der universellen Orientierung zu rauben, die ihnen das moderne Selbstbewußtsein einst versprach." (Bauman I995: 23) Diesem Paradox vermag sich vielleicht gerade eine nun fast schon klassisch selbstreflexive Wissenschaft wie die Ethnologie mit besonderer Kompetenz und Einfühlung zuzuwenden. 
https://doi.org/10.17104/9783406635991-183

Generiert durch IP '172.22.53.54', am 26.04.2023, 16:06:24.

Das Erstellen und Weitergeben von Kopien dieses PDFs ist nicht zulässig. 


\section{Methoden und Felder}

In der kulturwissenschaftlichen Forschung gibt es heute wenige methodische Zugänge und thematische Felder, von denen eine Europäische Ethnologie behaupten könnte, sie seien ausschließlich ihr Revier und Handwerkszeug. Sowohl in der historischen als auch in der Gegenwartsforschung haben sich in den letzten Jahrzehnten vielfältige Kooperations- wie Konkurrenzverhältnisse zu anderen Fächern ergeben, die scharfe disziplinäre Grenzziehungen schwierig, wenn nicht unmöglich machen. Selbst die alten Kanongegenstände der Volkskunde im Bereich historischer Volkskultur oder dörflicher Lebenswelt sind längst von anderen Fächern entdeckt.

So ist das Profil unseres Faches, jene kognitive Identität der Eigenund Fremdbilder, auch eher in bestimmten thematischen wie methodischen Zuschnitten zu suchen als in festen Reviermarkierungen. Es sind die spezifischen Fragestellungen und die spezifischen Wege, auf denen Antworten darauf gesucht werden, aus denen sich letztlich eine Handschrift ergibt, die - so scheint mir - sich in vieler Hinsicht immer noch kenntlich von der einer Kulturhistorikerin oder eines Kultursoziologen unterscheidet, die nominell dasselbe Thema bearbeiten. Denn diese thematische wie methodische Offenheit bedeutet keineswegs völlige Beliebigkeit. Gewiß sind wie in jedem anderen Fach immer wieder bestimmte Themen ,im Trend“, und es besteht eine Tendenz, sich für alles zuständig zu erklären. Doch legt man die Forschungsergebnisse unseres Faches nebeneinander, jene beeindrukkend lange Liste von Publikationen in Form von Magisterarbeiten und Dissertationen, von studentischen Studienprojekten und drittmittelgeförderten Forschungsprojekten, die in letzten zehn Jahren an Instituten für Volkskunde und für Europäische Ethnologie entstanden sind, dann zeichnen sich deutlich Themenstränge $a b$, die sich intern immer wieder bündeln und nicht nach außen hin auffasern.

Es sind thematische Linien, die nahe an die Lebenswelten und an die kulturelle Praxis der sozialen Akteure heranführen, die soziale Prozesse und Probleme in ihrer Entwicklung nachzeichnen, die gesellschaftliche Orte und Situationen in ihrer historischen Topographie wie in ihrer kulturellen Architektur darstellen, die nicht das statistisch Repräsentative, sondern das kulturell Signifikante und Charakteristische betonen, die den „harten“ Koordinaten „weiche“, differenzierte Schraffuren hinzufügen und die nicht zuletzt Hinweise darauf geben wollen, inwiefern das gewählte Thema neben dem wis- 